\title{
Die KRGÖ im Jahr 2015
}

Wie bereits im Geleitwort zu Heft 1 der BRGÖ 2015 berichtet, konstituierte sich die Kommission für Rechtsgeschichte Österreichs der Österreichischen Akademie der Wissenschaften (im Folgenden: KRGÖ-AW) am 14. Jänner 2015 neu und wählte Thomas OLECHOWSKI zu ihrem Obmann, Herbert KALB zum Obmann-Stellvertreter. Damit konnte eine nun schon über hundertfünfzigjährige Tradition rechtshistorischer Kommissionen an der ÖAW nach zweijähriger Unterbrechung wieder aufgenommen werden.

Die 2012 am Institut für Rechts- und Verfassungsgeschichte der Universität Wien gebildete „Abteilung KRGÖ“ (im Folgenden: KRGÖ-UW) unter der Leitung von OLECHOWSKI bleibt jedoch weiter bestehen, womit zugegebenermaßen ein Komplexitätsgrad erreicht ist, der auf den ersten Blick kafkaesk ${ }^{1}$ anmuten mag. Bei näherer Betrachtung ist die KRGÖ-AW jedoch wieder dort angekommen, wo sie bis 2003 war: Sie selbst verfügt weder über Personal noch Räume, wird aber in dieser Hinsicht vom Kommissionsobmann mitsamt seinen universitären Ressourcen unterstützt; im Gegensatz zur Periode bis 2003 erfolgt dies nunmehr ganz offiziell und gereicht hoffentlich beiden Seiten zum Vorteil.

Die „neue“ KRGÖ-AW präsentierte sich der Öffentlichkeit am 27. März mit einer Veranstaltung im Theatersaal der ÖAW. Nach einer Einführung durch den Obmann sprach der ehemalige Obmann-Stellvertreter emer.o.Univ.-Prof. Dr. Dr.h.c. Gernot Kocher Worte des Geden-

\footnotetext{
${ }^{1} \mathrm{Zu}$ Franz Kafka vgl. den Artikel von GANDORFER in BRGÖ 2014, 34-63.
}

kens für den am 13. Jänner verstorbenen langjährigen Obmann Werner OGRIS. Sodann wurden zwei Publikationen präsentiert, die aus dem an der KRGÖ durchgeführten FWF-Projekt „Die Wiener Rechts- und Staatswissenschaftliche Fakultät 1918-1938“" (P 21280) hervorgegangen sind: die gleichnamige Monographie von Thomas OlechOWSKI, Tamara EHS und Kamila StAUdigl-CieCHOWICZ wurde von Dr. Herbert POSCH vom Forum Zeitgeschichte der Universität Wien präsentiert; der BRGÖ-Band 2014/2 „Zwischen Wien und Czernowitz. Rechts- und Staatswissenschaftliche Karrierewege um 1918“, herausgegeben von STAUDIGL-CIECHOWICZ, EHS und OlechowsKI, erfuhr eine Präsentation durch Kommissionsmitglied und ObmannStellvertreter Herbert KALB.

Der MitarbeiterInnenstab der KRGÖ-UW hat sich im Laufe des vergangenen Jahres deutlich gewandelt: Frau Dr. Eva ORTLIEB ist mit 30. September, Herr DDr. Christoph SCHMETTERER mit 31. Dezember als Universitätsassistentin bzw. -assistent ausgeschieden; SCHMETTERER - der 2014 seine Rechtsanwaltsprüfung mit ausgezeichnetem Erfolg abgelegt hat - wird jedoch weiter als freier Dienstnehmer die Redaktion der BRGÖ leiten. Frau Katharina BERNOLD hat mit 23. Oktober eine Elternkarenzzeit angetreten, desgleichen Frau Mag. Kamila STAUDigLCiechowicz mit 24. Dezember, sodass der MitarbeiterInnenstab der KRGÖ-UW sich mit Stichtag 1. Jänner 2016 auf SCHMETTERER und auf Frau Mag. Laura Rosemarie RATHMANNER beschränkt, die seit 1. August 2015 als freie Dienstnehmerin für die KRGÖ-UW tätig ist. Nicht eingerechnet sind hier die beiden freien 
Mitarbeiterinnen Mag. Sandra WeISS und Mag. Susanne GMOSER, die nach wie vor auf Rechnung und im Auftrag der Akademie der Wissenschaften zu Göttingen am gemeinsamen Langzeitprojekt zur Erschließung der Akten des Reichshofrates arbeiten.

Das FWF-Projekt P 23747-G16 „Hans Kelsens Leben in Amerika (1940-1973) und die weltweite Verbreitung seiner Rechtslehre" wurde im Februar 2015 erfolgreich abgeschlossen; zwei Buchpublikationen, die aus diesem Projekt hervorgegangen sind, sollen 2016 erscheinen, die umfassende Biographie zu Hans Kelsen, an der OLECHOWSKI schreibt, ist weiter in Vorbereitung. Ebenfalls in Vorbereitung ist derzeit ein neues Forschungsprojekt, das eine umfassende rechtshistorische Aufarbeitung des Staatsvertrages von St. Germain-en-Laye vom 10. September 1919 zum Gegenstand hat.

Die KRGÖ war im Jahr 2015 Mitveranstalterin bzw. Veranstalterin zweier Tagungen:

Vom 17. bis 20. Juni 2015 fand die Tagung „Der Wiener Kongress 1814/15. Politische Kultur und internationale Politik" statt; Mitveranstalter waren neben der KRGÖ-UW die ÖAW, die Kommission für Neuere Geschichte Österreichs, die Wiener Vorlesungen sowie die Universitäten Innsbruck und Klagenfurt. Tagungsorte waren der Theatersaal der ÖAW, das ehemalige Palais Metternich (nunmehr Botschaft der Republik Italien), die ehemalige Haus-, Hof- und Staatskanzlei (nunmehr Bundeskanzleramt) sowie das Wiener Rathaus. Die 34 Vorträge widmeten sich den Netzwerken und Akteuren, den Verhandlungen von ihrer rechtlichen und politischen
Seite, dem Wiener Kongress als Ort kultureller Produktion wie auch dem Nachleben des Kongresses in der Historiographie. Eine Publikation der Beiträge wird 2016 in der Buchreihe „Internationale Geschichte“ im Verlag der ÖAW erfolgen.

Vom 11. bis zum 12. Dezember 2015 veranstaltete die KRGÖ (KRGÖ-AW und KRGÖ-UW) die Tagung "Gerichtsvielfalt in Wien. Forschungen zum modernen Gerichtsbegriff". 18 Referentinnen und Referenten sprachen im Theatersaal der ÖAW zu verschiedenen, teils historischen, teils heute noch existierenden Gerichten, gerichtsähnlichen Behörden und sonstigen rechtsprechenden Organen mit Sitz in Wien. Eine Publikation wird in Heft 2 der BRGÖ 2016 erfolgen; siehe dort für weitere Details.

Der traditionell an dieser Stelle erfolgende Bericht über das Budget der KRGÖ erfolgt ab nun sinnvollerweise für KRGÖ-AW und KRGÖ-UW gemeinsam: Von der Universität Wien erhielt die KRGÖ-UW ein Personal- und Sachmittelbudget in der Höhe von $€ 159.893,25$; davon wurden $€ 110.286,56$ aufgebraucht, der Rest in Rücklagen umgewandelt. Von der ÖAW kam ein Sachmittelbudget in Höhe von $€ 2.000$,-. An Drittmittel sind zu nennen: $€ 35.692,44$ von der Akademie der Wissenschaften zu Göttingen, $€ 11.000$,- vom FWF und $€ 6.733,60$ von sonstigen Fördergebern. Wertet man nun die Gelder der Universität Wien als „Erstmittel“, jene der ÖAW als "Zweitmittel" und die übrigen als "Drittmittel“, so ergibt sich daraus ein Drittmittelanteil von $32,2 \%$.

Thomas OLECHOWSKI 